\title{
Digitization of the FBS: Its Future Use and Expected Results
}

\author{
Areg M. Mickaelian \\ Byurakan Astrophysical Observatory (BAO), Byurakan 378433, \\ Armenia, and Isaac Newton Institute of Chile, Armenian Branch. \\ E-mail: aregmick@bao.sci.am
}

\begin{abstract}
The First Byurakan Survey (FBS) is the largest spectral survey in the Northern sky. Its plates contain low-dispersion spectra for some $20,000,000$ objects. The FBS spectra allow selection of objects by their color, broad emission or absorption lines, or SED; to discover, classify and investigate them. The FBS was conducted originally to search for UVX galaxies (1500 Markarian galaxies were discovered). Selection of blue stellar objects, red stars, and identification of IRAS sources have also been done by means of the FBS spectra. All these projects have been carried out by visual inspection of the plates. Digitization will give new possibilities to search for many new objects: new bright QSOs $\left(\mathrm{m}<18^{m}\right)$, new Markarian (UVX) galaxies, BCDGs, optical counterparts of IR, radio, $\mathrm{X}$-ray and other sources, late-type stars, planetary nebulae, emission-line stars, and white dwarfs; and to study star clusters and clusters of galaxies. The digitized FBS will be available via the Internet and on CDs. Software and an appropriate interface for working with the data will be provided.
\end{abstract}

\section{Introduction}

The Digitized Sky Survey (DSS) is widely used by all astronomers. It gives good astrometric, photometric and morphological data on all the objects with $\delta>-30^{\circ}$ to $21^{m}$. However, it cannot give more information on the nature of the objects. Such information is provided by spectroscopic data, but unfortunately, there isn't any all-sky spectroscopic survey. The Hamburg Survey was digitized recently, giving low-dispersion spectra for objects to $18^{m}$ at high galactic latitudes $\left(|b|>20^{\circ}\right)$ (Engels et al. 2001). The best data for an area of $10,000 \mathrm{deg}^{2}$ of the Northern Sky will be provided by the Sloan Digital Sky Survey (SDSS). However, at present there is the possibility to provide such information to $17.5^{m}-18^{m}$. The First Byurakan Survey (FBS) is a large spectral survey, covering $17,000 \mathrm{deg}^{2}$ of all the Northern Sky and part of the Southern Sky at high galactic latitudes (Markarian et al. 1989). It provides unique observational material for discovery, classification and investigation of various types of objects.

FBS low-dispersion spectra are rather useful for selection of objects by different criteria (such as their color, broad emission or absorption lines, spectral energy distribution), quick study of separate fields for the content of different 
types of objects, etc. The number and classes of new objects discovered in the FBS emphasize the necessity for open, free access to this information for the astronomical community.

As many astronomers are engaged in searching for new objects and identification of different sources, the FBS low-dispersion spectra may be useful for such work for better understanding of their nature, before making detailed investigations with higher dispersions and resolutions. There is a need for access to this database for all the astronomical community. Digitized information may be widely distributed on CDs, as well as by the Internet. For this reason it is desirable first to create the digitized copies of the FBS plates with a high resolution.

This project will allow use of the First Byurakan Survey together with the DSS for selection, classification and investigation of many new interesting objects, as well as analysis of sample composition in definite areas of interest combining all the data in a given region. The FBS will provide a unique database of homogeneous data on some $20,000,000$ objects at high galactic latitudes.

\section{Description of the $F B S$ and its Low-Dispersion Spectra}

The First Byurakan Survey was carried out by B.E. Markarian, V.A. Lipovetsky and J.A. Stepanian in 1965-1980 with the Byurakan Observatory 102/132/213 $\mathrm{cm}\left(40^{\prime \prime} / 52^{\prime \prime} / 84^{\prime \prime}\right)$ Schmidt telescope using the $1.5^{\circ}$ prism (Markarian et al. 1989). 2050 Kodak IIAF, IIaF, IIF, and 103aF photographic plates in 1133 fields $\left(4^{\circ} \times 4^{\circ}\right.$ each, the size being $\left.16 \mathrm{~cm} \times 16 \mathrm{~cm}\right)$ were taken. The FBS covers $17,000 \mathrm{deg}^{2}$ of all the Northern sky and part of the Southern sky $\left(\delta>-15^{\circ}\right)$ at high galactic latitudes $\left(|b|>15^{\circ}\right)$. In some regions, it even goes down to $\delta=-19^{\circ}$ and $|b|=10^{\circ}$. The limiting magnitude on different plates varies in the range of $16.5-19.5$ in $\mathrm{V}$, however for the majority it is $17.5^{m}-18^{m}$. The scale is $96.8^{\prime \prime} / \mathrm{mm}$ and the dispersion is $1800 \AA / \mathrm{mm}$ near $\mathrm{H} \gamma$ and $2500 \AA / \mathrm{mm}$ near $\mathrm{H} \beta$ (mean spectral resolution being about $50 \AA$ ). Low-dispersion spectra cover the range 3400-6900 $\AA$, and there is a sensitivity gap near $5300 \AA$, dividing the spectra into red and blue parts. It is possible to compare the red and blue parts of the spectrum (easily separating red and blue objects), follow the spectral energy distribution, notice some emission and absorption lines (such as broad Balmer lines, molecular bands, He, [OIII $\mathrm{N}_{1}+\mathrm{N}_{2}$ and [OII] lines; broad emission lines of QSOs and Seyferts, etc.), thus gaining some understanding about the nature of the objects (Fig. 1). The FBS is made up of zones (strips), each covering $4^{\circ}$ in declination and all right ascensions except the Galactic plane regions. In all there are 28 zones, which are named by their central declination (e.g., zone $+27^{\circ}$ covers $+25^{\circ}<\delta<+29^{\circ}$, zone $+63^{\circ}$ has $+61^{\circ}<\delta<+65^{\circ}$, etc). The zones and the neighboring plates in right ascension overlap about $0.1^{\circ}$ (as the exact size of a plate is $4.1^{\circ} \times 4.1^{\circ}$ ) thus making the whole area complete. Each FBS plate contains low-dispersion spectra of some 15,000-20,000 objects, and there are some $20,000,000$ objects in the whole survey. 


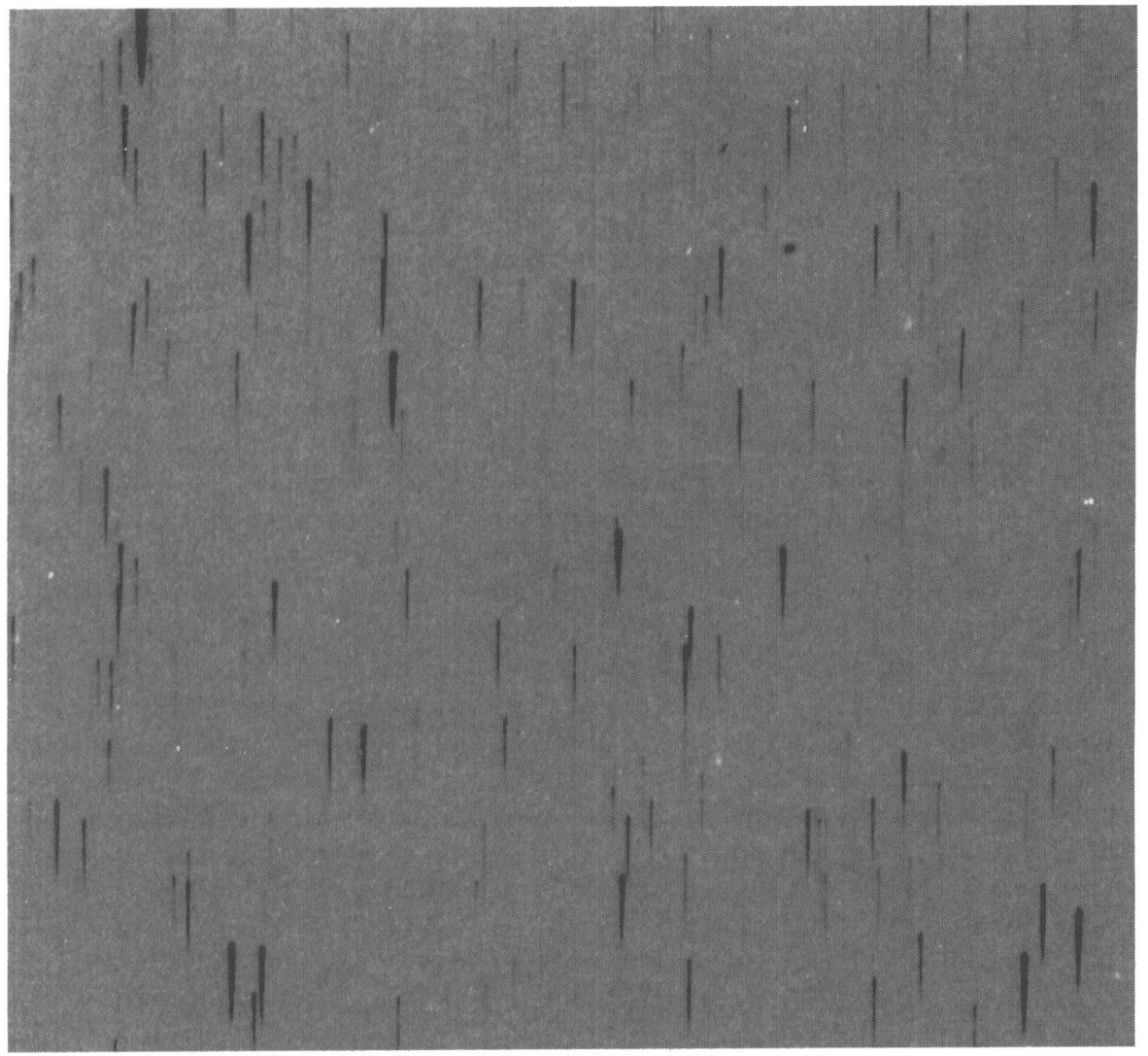

Figure 1. A piece of an FBS plate with its low-dispersion spectra. 


\section{Results Obtained on the Basis of the FBS Plates}

\subsection{Markarian Galaxies}

The FBS was conducted originally to search for galaxies with UV-excess. The discovery of 1515 UV-excess (UVX) galaxies by Markarian and colleagues (later called Markarian galaxies) was the first and the most important work based on the FBS plates (Markarian 1967; Mazzarella \& Balzano 1986; Markarian et al. 1989). Spectral observations of these objects were carried out first by Weedman \& Khachikian (1968), and by Arakelian, Dibay \& Esipov (1970), and then by hundreds of other authors. The study of Markarian galaxies led to the discovery of many new Seyferts and the first spectral classification of this type of object (Weedman \& Khachikian 1971). Among them, there are more than 200 Seyferts, dozens of QSOs, 17 LINERs, a few hundred starburst and isolated HII galaxies, $3 \mathrm{BL}$ Lac objects, radio, IR, X- and gamma-ray sources, interacting and merging objects, and galaxies with double and multiple nuclei. During the last 30 years, more than 3500 scientific papers have been published on the study of these objects. There are many Markarian galaxies, which are the subject of study for understanding the AGN phenomenon, starburst activity and evolution of galaxies, high-luminosity IR radiation, AGN variability, double and multiple structure of the nucleus, composite spectrum AGNs, galaxy interactions and merging, connections between different types of active galaxies, early stages of evolution of galaxies, and other important topics of modern extragalactic astronomy. Some Markarian galaxies are crucial for solving different important problems: Mrk 231 is the most luminous infrared galaxy (ULIG) in the Local Universe (e.g., Weedman 1999); some Markarian galaxies are blue compact dwarf galaxies (BCDGs), such as the remarkable BCD galaxy Mrk 116 (I $\mathrm{Zw} \mathrm{18}$ ), the most metal-deficient BCDG (most of the known objects of this type are Markarian and SBS galaxies (Second Byurakan Survey; Markarian \& Stepanian 1983)); Mrk 501 is one of the 5 known TeV sources; Mrk 938 is the first dynamical merger discovered observationally; Mrk 766 is one of the famous NLS1 galaxies; Mrk 273 is a wonderful double-double nuclei galaxy; Mrk 6 shows variations of spectral lines typical of different types of objects and very high column density of $H$ in X-rays; Mrk 421 and Mrk 501 (BL Lac objects) are among the strongest $\mathrm{X}$-ray sources; Mrk 926 is one of the rare Sy1 galaxies having LINER properties (like 3C 390.3); Mrk 266 has a multiple structure nuclear region; Mrk 110 is crucial for understanding differences between NLS1s and BLS1s; Mrk 231 and Mrk 507 are among the 5 known superstrongest FeII emitters (ex. Véron-Cetty \& Véron 2000); Mrk 530, Mrk 993 and Mrk 1018 change their spectra from Sy2 to Sy1 thus being important for the unified scheme of AGN. The Markarian survey was the first systematic survey for AGNs.

\section{2. $\quad F B S$ Stellar Objects}

The second part of the FBS was devoted to the discovery and investigation of blue (UVX) stellar objects. It was carried out by H.V. Abrahamian and A.M. Mickaelian in 1987-1996 (Abrahamian \& Mickaelian 1996; Mickaelian 1994, and references therein) in 278 FBS fields, in a $4009 \mathrm{deg}^{2}$ area of the FBS $\left(+33^{\circ}<\delta<+45^{\circ}\right.$ and $\left.+61^{\circ}<\delta<+90^{\circ}\right)$. The main purpose of this work was discovery of new bright QSOs (as Markarian's survey was aimed only at extended 
objects), Seyferts, other compact galaxies, planetary nebula nuclei, cataclysmic variables (CV), white dwarfs (WD), subdwarfs, and other peculiar stellar objects. This project is similar to the Palomar-Green survey (Green et al. 1986), however it was done on the basis of the low-dispersion spectra. 1103 objects have been selected, including 716 new blue stellar objects and many new objects were revealed. Subsamples of candidate QSOs, WDs, CVs, continuous spectrum objects (possible BL Lacs and DCs) have been made up and observations have been carried out for spectral identification of these objects. 40 new bright AGNs have been discovered already among them (Mickaelian et al. 2001a; Véron-Cetty \& Véron 2001), proving that the FBS can provide data for enriching our knowledge of the Local Universe. The local density of QSOs and the completeness of the Bright Quasar Survey (BQS) (Schmidt \& Green 1983) have been re-estimated. Moreover, cross-correlations of NVSS/USNO and ROSAT/USNO objects, and checking their low-dispersion spectra in the FBS with further follow-up spectroscopy identified new QSOs in the FBS plates which had not been selected before. A number of interesting WDs and CVs have been discovered too, such as the new bright SW Sex type cataclysmic variable (Mickaelian et al. 2001b). In all, 11 lists of the 2nd part of the FBS have been published. The full catalog of the FBS blue stellar objects is available at CDS (Abrahamian et al. 1999).

A survey for late-type stars on the FBS plates has been carried out since 1987 by H.V.Abrahamian and K.S.Gigoyan (Gigoyan et al. 2001 and references therein). 813 late M-type and carbon stars have been selected already in an area of $6640 \mathrm{deg}^{2}$ in 14 zones of the FBS, including 560 new ones. Surveys for carbon stars at high galactic latitudes are very rare. Discovery and study of such objects is necessary for the study of the kinematics and chemical composition of the Galactic halo. Besides, many objects with extended dust shells, as well as two new extremely rare dwarf carbon stars have been discovered. Twelve lists of FBS late-type stars have been published.

\subsection{Identifications of IRAS Sources}

Work on the selection of galaxies, blue stellar objects (including QSOs and Seyferts) and late-type stars led to a new program of optical identifications of IRAS sources on the basis of the FBS plates (Mickaelian 1995). Since 1995, 1577 previously unidentified IRAS point sources (IRAS 1988, 1989) have been optically identified in the zone $+61^{\circ}<\delta<+90^{\circ}$ with a surface area of 1487 $\mathrm{deg}^{2}$. In this area, FBS plates have better limiting magnitudes (on average: 18.1 in V). 1178 of the identified sources are galaxies. The identification program produced two samples of objects: BIS (Byurakan-IRAS Stars), and BIG (Byurakan-IRAS Galaxies). Seven lists of galaxies (Mickaelian 2001, and references therein) and 5 lists of stars (Mickaelian \& Gigoyan 2001, and references therein) have been published already. The BIS and BIG objects are present in many databases, including CDS catalogs, SIMBAD and NED (extragalactic objects). These samples are the subjects of multi-faceted investigations. $\mathrm{Ob}-$ servations revealed many interesting objects, including Seyferts, LINERs and composite spectrum AGNs (e.g. Balayan et al. 2001). The IRAS galaxy sample contains AGNs, high-luminosity IR galaxies (LIGs, ULIGs, HLIGs, Sanders \& Mirabel 1996), groups of galaxies (including compact ones), interacting and merging galaxies, etc. Some 30 IRAS sources showing galaxy colors have no opti- 
cal counterparts even in the DSS fields, being new candidates for obscured IRAS galaxies (probable ULIGs) and important for revealing the real IR galaxy population. Study of the new sample of IRAS galaxies, especially the high-luminosity IR galaxies, may lead to understanding of the interrelationship between AGN and starburst activity induced by galaxy interactions and merging. The subsample of galactic objects is also interesting, as it contains new planetary nebulae, AGB stars, late M and carbon stars. Some of them have IR excesses, suggesting extended dust shells; in addition, a number of stars show evidence of variability.

Table 1 presents a summary of projects undertaken on the basis of the FBS observational material with brief data: name of the project, years, main authors, area of investigation, number of objects involved, and references.

Table 1. Projects Undertaken on the Basis of the FBS

\begin{tabular}{|c|c|c|c|c|c|}
\hline Project & Years & Authors & $\begin{array}{r}\text { Area } \\
\left(\mathrm{deg}^{2}\right)\end{array}$ & Objects & Ref \\
\hline $\begin{array}{l}\text { UVX galaxies } \\
\text { (Markarian galaxies) }\end{array}$ & $1965-1980$ & $\begin{array}{l}\text { Markarian, } \\
\text { Lipovetsky, } \\
\text { Stepanian }\end{array}$ & 17000 & 1515 & (1) \\
\hline $\begin{array}{l}\text { UVX stars } \\
\text { (2nd part of the FBS) }\end{array}$ & $1987-1996$ & $\begin{array}{l}\text { Abrahamian, } \\
\text { Mickaelian }\end{array}$ & 4009 & 1103 & (2) \\
\hline Late-type stars & $1987-2001$ & $\begin{array}{l}\text { Abrahamian, } \\
\text { Gigoyan }\end{array}$ & 6640 & 813 & (3) \\
\hline IRAS galaxies (BIG) & $1995-2001$ & Mickaelian & 1487 & 1178 & (4) \\
\hline IRAS stars (BIS) & $1995-2001$ & $\begin{array}{l}\text { Mickaelian, } \\
\text { Gigoyan }\end{array}$ & 1487 & 370 & (5) \\
\hline
\end{tabular}

References: (1) Markarian et al. 1989; (2) Abrahamian \& Mickaelian 1996; (3) Gigoyan et al. 2001; (4) Mickaelian 2001; (5) Mickaelian \& Gigoyan 2001.

\section{What Information is Still in the Plates?: New Possibilities}

Some five thousand interesting objects have already been found in the First Byurakan Survey plates. Nevertheless, its observational material is still useful for new studies. If digitized, FBS low-dispersion spectra can offer the possibility of searching for many new objects, such as:

- New bright QSOs $\left(\mathrm{m}<18^{m}\right)$. As the visual selection has missed many objects, and creation of numerical criteria for selection of candidate QSOs will be much more efficient, one can expect some 200 new bright QSOs $\left(\mathrm{m}<16.1^{m}\right.$, for a comparison with the BQS) from the FBS, and some 1000 
fainter objects, and it will be the most complete sample for study of their local density;

- New Markarian (UVX) galaxies. The same situation applies for UVX galaxies, as visual selection has missed objects near the limits of the plates. It is necessary to have 1-D records for all Markarian galaxies to be able to continue a homogeneous selection of such objects;

- New blue stellar objects. The 2nd part of the FBS will be continued for the whole area and a total number of some 4500 objects is expected. The techniques of selection and preliminary classification have been worked out during 1987-1996 and the digitization will make possible quicker and better quality completion;

- New BCDGs. 22 Markarian galaxies and 135 SBS galaxies are candidate BCDGs. These two surveys have provided the vast majority of the sample of these objects. A search for compact UVX galaxies in the FBS will provide more BCDGs, making possible the understanding of the early stages of galaxy evolution;

- Optical counterparts of IR, radio, X-ray and other sources (optical identifications). The program of identification of IRAS sources proved that this kind of work can be very efficient with the help of low-dispersion spectra. The Hamburg/ROSAT identification program (Bade et al. 1998) comes to prove again the importance of such work on the basis of low-dispersion spectra. The digitization will allow continuation of the program of IRAS identifications to other regions of the FBS, as well as identifications for radio and X-ray sources, too;

- Late-type stars (M, S, carbon stars). The faintest late-type stars look like dots on the low-dispersion plates. It is rather low confidence to select such objects, as they may appear to be defects as well. For automatic selection it will be easier to separate real spectra from artificial ones;

- Planetary nebulae. There still may be faint undetected planetary nebulae. As their number approaches the limit, it seems interesting to find out all PNs in the Galaxy, particularly at high galactic latitudes;

- All emission-line stars (CV, etc). Their emission lines are sometimes of low contrast with respect to the continuum. That is why one needs to deal with 1-D spectral cuts and computer analysis for better selection;

- Non-UVX white dwarfs. During the completion of the Second part of the FBS, the authors have noticed that there are a lot of white dwarfs without UVX. Many such objects have been marked but not selected. However, they may be picked out easily by their broad absorption lines and complement the sample of high-latitude WDs for further studies of stellar evolution and evolution of the Galactic halo;

- Studies of star clusters and clusters of galaxies. It is rather convenient to study clusters using homogeneous observational material, especially having 
the low-dispersion spectra of all the objects. Estimates of the distribution of brightness, colors and types of objects may be made. Rough estimate of redshifts (membership in the clusters) for galaxies may be made, too.

The FBS will serve as a database for the astronomical community via the Internet, and many new tasks may be put forward by other astronomers, too.

\section{Technical Description of the Program of Digitization}

Taking into account the size of the photographic grains $(25-30 \mu \mathrm{m})$, it is reasonable to scan the plates with a resolution of some $10 \mu \mathrm{m}$ (about $2500 \mathrm{dpi}$ ). Therefore, it is planned to scan the FBS plates with a scanner with a resolution of $2400 \mathrm{dpi}$ (for quick completion of the project). The files containing the scanned plates at first will be saved on HDD. For this, large volumes of HDD are needed. Corresponding software for giving coordinate information, the possibility of comparing with the corresponding DSS fields, making 1-D cuts for each spectrum, etc. will be created. Some 170 pixels of data will be obtained for each FBS spectrum (the length of each FBS spectrum is $1700 \mu \mathrm{m}$ ).

It will be useful to offer users a numerical classification scheme. Classification schemes based on criteria worked out during the selection of blue stellar objects and identification of IRAS sources will be developed. The classification principles are based on the relation of magnitudes and widths of the spectra (for separation of stellar and diffuse objects), spectral energy distribution (color), and presence or absence of broad spectral lines. Classification will be linked to general classification schemes using standard objects in the fields. We plan also to make some rough photometric calibration using photometric standards to make possible a quick estimate of magnitudes from the FBS. It is estimated that up to $0.3^{m}$ accuracy may be reached. However, we don't pursue photometric precision. It should be done using the DSS database (rough photometry by APS) (Pennington et al. 1993).

Each spectrum will be presented as a small table (of 170 lines), and each (FBS) plate contains some 20,000 such spectra. Taking into account the difficulties concerning the use of full image data to identify and measure objects while dealing with an FBS plate, it makes sense to create a catalog of objects with all available data (positional, photometric, and spectral), and recommend it to users, too, for quicker access to the data.

In all (if we digitize all the plates) 40,000,000 spectra from the FBS plates, or (if we digitize only the best plates in each field) 20,000,000 FBS spectra are to be recorded. Data from each plate will be about $450 \mathrm{Mb}$, and after archiving - some $45 \mathrm{Mb}$. Appropriate media are necessary to store the large volumes of data from the digitized images. CD-ROMs are the most convenient and cheap units for this purpose. Therefore the data from the full plate scans, the 1-D spectra, the catalog of objects, and corresponding software will be copied onto CDs. Data for 14 plates (45Mb each) will be written on each CD (650Mb). In all, 81 CDs will be needed for the FBS database (in the case of digitization of all the plates - 147). The whole FBS database will take some 50Gb.

A web page for the FBS will be created and all the information will be available to astronomers. It will also be possible to extract separately 1-D spec- 
tra of objects by searching for them in the FBS catalog (like APS for the DSS), without taking larger volumes of information for the corresponding fields. Users will be able to compare the fields and data with corresponding ones from other surveys, too, including DSS, the Hamburg Survey, etc. All this will make it very efficient to work with unknown objects, especially for the people working in extragalactic astronomy.

By our estimates, the FBS database will be available at the end of 2002 or at the beginning of 2003 .

\section{References}

Abrahamian, H.V., \& Mickaelian, A.M. 1996, Ap, 39, 531

Abrahamian, H.V., Mickaelian, A.M., Lipovetsky, V.A., \& Stepanian, J.A. 1999, Catalog No.II/223 at CDS, Strasbourg, at http://vizier.u-strasbg.fr/cgibin/VizieR?-source=II/223/fbs2

Arakelian, M.A., Dibay, E.A., \& Esipov, V.F. 1970, Afz, 6, 39

Bade, N., Engels, D., Voges, W. et al. 1998, A\&AS, 127, 145

Balayan, S.K., Hakopian, S.A., Mickaelian, A.M., \& Burenkov, A.N. 2001, AstL, 27,635

DSS web page at STScI: http://stdatu.stsci.edu/dss/

Engels, D. et al. 2001, Proceedings of the Conference: The New Era of WideField Astronomy, ASP Conf. Series, Vol. 232, Eds. R.G. Clowes, A.J. Adamson, \& G.E. Bromage (in press)

Gigoyan, K.S., Abrahamian, H.V., Azzopardi, M., \& Russeil, D. 2001, Ap, 44, 88

Green, R.F., Schmidt, M., \& Liebert, J. 1986, ApJS, 61, 305

IRAS Catalogs and Atlases 1988, 2. The Point Source Catalog. Declination $90^{\circ}>\delta>30^{\circ}$, Joint IRAS Science Working Group, NASA, Washington, DC: US GPO

IRAS Faint Source Catalog 1989, $|b|>10^{\circ}$, Version 2, On Optical Disc, "Selected Astronomical Catalogs", Supplied by NASA, Vol. 1

Markarian, B.E. 1967, Ap, 3, 55

Markarian, B.E., \& Stepanian, J.A. 1983, Ap, 19, 639

Markarian, B.E., Lipovetski, V.A., Stepanian, J.A., Erastova, L.K., \& Shapovalova, A.I. 1989, Commun. Special Astrophys. Obs., 62, 5

Mickaelian, A.M. 1994, Discovery and Investigation of the Blue Stellar Objects of the First Byurakan Survey, Ph.D. Thesis, Byurakan, 284 p.

Mickaelian, A.M. 1995, Ap, 38, 349

Mickaelian, A.M. 2001, Ap, 44, 185

Mickaelian, A.M., \& Gigoyan, K.S. 2001, Ap, 44, 222

Mickaelian, A.M, Gonçalves, A.C., Véron-Cetty, M.P., \& Véron, P. 2001a, Ap, 44,14

Mickaelian, A.M., Balayan, S.K., Ilovaisky, S.A., Chevalier, C., Véron-Cetty, M.P., \& Véron, P. 2001b, astro-ph/0108377, OHP Preprint No. 155 
Pennington, R.L., Humphreys, R.M., Odewahn, S.C., Zumach, W., \& Thurmes, P.M. 1993, PASP, 105, 521

Sanders, D.B., \& Mirabel, I.F. 1996, ARA\&A, 34, 749

Schmidt, M., \& Green, R.F. 1983, ApJ, 269, 352

SDSS web page at Johns Hopkins Univ.: http://tarkus.pha.jhu.edu/

Véron-Cetty, M.-P., \& Véron, P. 2000, A\&ARv, 10, 81

Véron-Cetty, M.-P., \& Véron, P. 2001, A\&A, 374, 92; and the electronic version of the Catalog of QSOs (10th edition) at http://cdsweb.u-strasbg.fr/cgibin/qcat?J/A+A/374/92 or http://www.obs-hp.fr

Weedman, D.W. 1999, Proceedings of the IAU Symp. 194: Active Galactic Nuclei and Related Phenomena, Eds. Terzian Y., Weedman D., Khachikian E., Astron. Soc. Pacific, 191

Weedman, D.W. \& Khachikian, E.Ye., 1968, Afz, 4, 587

Weedman, D.W. \& Khachikian, E.Ye., 1971, Ap, 7, 389 